\title{
Fixed Point Approximation of Monotone Nonexpansive Mappings in Hyperbolic Spaces
}

\author{
Amna Kalsoom, ${ }^{1}$ Naeem Saleem (D), ${ }^{2}$ Hüseyin Ișık $\left(\mathbb{D},{ }^{3}\right.$ Tareq M. Al-Shami ${ }^{1},{ }^{4}$ Amna Bibi, ${ }^{1}$ \\ and Hafsa Khan ${ }^{1}$ \\ ${ }^{1}$ Department of Mathematics and Statistics, International Islamic University, Islamabad, Pakistan \\ ${ }^{2}$ Department of Mathematics, University of Management and Technology, Lahore, Pakistan \\ ${ }^{3}$ Department of Engineering Science, Bandırma Onyedi Eylül University, Bandırma 10200, Balıkesir, Turkey \\ ${ }^{4}$ Department of Mathematics, Sana'a University, Sana'a, Yemen
}

Correspondence should be addressed to Hüseyin Işı; huseyin.isik@tdtu.edu.vn

and Tareq M. Al-Shami; tareqalshami83@gmail.com

Received 10 May 2021; Revised 1 July 2021; Accepted 13 July 2021; Published 10 August 2021

Academic Editor: Mustafa Avci

Copyright ( 2021 Amna Kalsoom et al. This is an open access article distributed under the Creative Commons Attribution License, which permits unrestricted use, distribution, and reproduction in any medium, provided the original work is properly cited.

Fixed points of monotone $\alpha$-nonexpansive and generalized $\beta$-nonexpansive mappings have been approximated in Banach space. Our purpose is to approximate the fixed points for the above mappings in hyperbolic space. We prove the existence and convergence results using some iteration processes.

\section{Introduction}

In 1965, Browder [1], Göhde [2], and Kirk [3] started working in the approximation of fixed point for nonexpansive mappings. Firstly, Browder obtained fixed point theorem for nonexpansive mapping on a subset of a Hilbert space that is closed bounded and convex. Soon after, Browder [1] and Göhde [2] generalized the same result from a Hilbert space to a uniformly convex Banach space. Kirk [3] utilized normal structure property in a reflexive Banach space to sum up the similar results. Recently, Dehici and Najeh [4] and Tan and Cho [5] approximated fixed point result for nonexpansive mappings in Banach space and Hilbert space.

Fixed point theory in partially ordered metric spaces has been initiated by Ran and Reurings [6] for finding application to matrix equation. Nieto and Lopez [7] extended their result for nondecreasing mapping and presented an application to differential equations. Recently, Song et al. [8] extended the notion of $\alpha$-nonexpansive mapping to monotone $\alpha$-nonexpansive mapping in order Banach spaces and obtained some existence and convergence theorem for the Mann iteration (see also [9] and the reference therein). Motivated by the work of Suzuki [10], Aoyama and Kohsaka [11],
Dehaish and Khamsi [9], and Song et al. [8], Pant and Shukla obtained existence results in ordered Banach space for a wider class of nonexpansive mappings [12, 13]. There are many mathematicians who worked on weak and strong convergence of nonexpansive mappings and its generalizations by using one step, two step, and multistep iteration process $([8,14,15])$. We obtain existence results in partial ordered hyperbolic space for monotone generalized $\alpha$-nonexpansive and monotone generalized $\beta$-nonexpansive map. Particularly, in Section 3, some auxiliary results and existence theorems for monotone $\alpha$-nonexpansive mappings in ordered hyperbolic spaces are presented. In Section 4, we presented numerical examples and graphical representation. In Section 5 , we obtained some existence results for monotone generalized $\beta$-nonexpansive mappings in ordered hyperbolic spaces.

\section{Preliminaries}

In 1976, the concept of $\Delta$-convergence was given by Lim [14]. Lim [14] initiated the idea that in a metric space, $\Delta$ -convergence is possible. This concept is adapted for CAT(0) spaces by Kirk and Panyanak [16], and they have indicated that in numerous Banach space, outcomes 
comprising weak convergence were having exactly accurate analogs in this manner.

Definition 1. A self map $\mathscr{T}$ on $W$ is known as Lipschitz, if there exists $k \geq 0$ such that

$$
\sigma(\mathscr{T} \xi, \mathscr{T} \eta) \leq k \sigma(\xi, \eta) \text {, for all } \xi, \eta \in W
$$

$\mathscr{T}$ is called to be contractive if $k \in(0,1]$, and $\mathscr{T}$ is called nonexpansive mapping, if $k=1$, that is,

$$
\sigma(\mathscr{T} \xi, \mathscr{T} \eta) \leq \sigma(\xi, \eta) \text {, for all } \xi, \eta \in W
$$

Suzuki [10] introduced an interesting generalized nonexpansive mapping as follows.

Definition 2. A self map $\mathscr{T}$ on $W$ is said to satisfy condition (3), if for all $\xi, \eta \in W$,

$$
\frac{1}{2} \sigma(\xi, \mathscr{T} \xi) \leq \sigma(\xi, \eta) \Rightarrow \sigma(\mathscr{T} \xi, \mathscr{T} \eta) \leq \sigma(\xi, \eta)
$$

Suzuki type generalized nonexpansive mapping is another name of self map $\mathscr{T}$ holding condition (3).

Many generalizations of nonexpansive mapping have been introduced in the literature (see [17-19]). Aoyama and Kohsaka [11] defined a new type of nonexpansive mapping that satisfies the condition (3) known as $\alpha$-nonexpansive mapping as follows.

Definition 3. Let $W$ be a nonempty subset of a Banach space $H$. A self map $\mathscr{T}$ on $W$ can be referred $\alpha$-nonexpansive mapping, if for all $\xi, \eta \in W$ and $\alpha<1$,

$$
\|\mathscr{T} \xi-\mathscr{T} \eta\|^{2} \leq \alpha\|\mathscr{T} \xi-\eta\|^{2}+\alpha\|\xi-\mathscr{T} \eta\|^{2}+(1-2 \alpha)\|\xi-\eta\|^{2} .
$$

The concept of monotone nonexpansive mappings was introduced in 2015 by Bachar and Khamsi [20], and they studied common approximate fixed point of monotone nonexpansive semigroup. To determine some order fixed points, Dehaish and Khamsi [9] proposed weak convergence theorems of the Mann iteration process for monotone nonexpansive mappings in uniformly convex ordered Banach spaces.

Definition 4. Let $T$ be a self map on $W$; then, $T$ is said to be

(1) monotone [20] if $T \xi \preceq T \eta$ for all $\xi, \eta \in W$ with $\xi \preceq \eta$;

(2) monotone nonexpansive [20] if $T$ is monotone and

$$
\sigma(T \xi, T \eta) \leq \sigma(\xi, \eta)
$$

for all $\xi, \eta \in W$ with $\xi \preceq \eta$;

(3) monotone quasi-nonexpansive [8] if $T$ is monotone and

$$
\sigma(T \xi, \eta) \leq \sigma(\xi, \eta)
$$

where $\xi \in W$ and $\eta \in F(T)$, and $F(T)$ is the set of fixed points.

Definition 5 [21]. $(H, \sigma, S)$ is called a hyperbolic space, if ( $H, \sigma)$ is a metric space and $S: H \times H \times[0,1] \longrightarrow H$ is a function holding

(i) $\sigma(\zeta, S(\xi, \eta, \lambda)) \leq(1-\lambda) \sigma(\zeta, \xi)+\lambda \sigma(\zeta, \eta)$;

(ii) $\sigma\left(S\left(\xi, \eta, \lambda_{1}\right), S\left(\xi, \eta, \lambda_{2}\right)\right) \leq\left|\lambda_{1}-\lambda_{2}\right| \sigma(\xi, \eta)$;

(iii) $S(\xi, \eta, \lambda)=S(\eta, \xi, 1-\lambda)$;

(iv) $\sigma(S(\xi, \zeta, \lambda), S(\eta, \nu, \lambda)) \leq(1-\lambda) \sigma(\xi, \eta)+\lambda \sigma(\zeta, \nu)$,

for all $\xi, \eta, \zeta, v \in H$ and $\lambda, \lambda_{1}, \lambda_{2} \in[0,1]$.

Here, (i) defines the convexity in metric space $(H, \sigma)$ that was first considered by Takahashi [22], (ii) provides a unique geodesic between any two elements $\xi$ and $\eta$ of $H$ by convexity map $S$ that is the space of hyperbolic type in the sense of Goebel and Kirk [23], (iii) provides symmetry along the direction of geodesic, and (iv) defines negative curvature or hyperbolicity of metric space was first considered by Itoh [24].

Theorem 6 [11]. Let $W$ be a nonempty closed convex subset of a uniformly convex Banach space $H$ and $\mathscr{T}: W \longrightarrow W$ be an $\alpha$-nonexpansive mapping. Then, $F(\mathscr{T})$ is nonempty iff there exists $\xi \in W$ such that $\left\{T^{n}(\xi)\right\}$ is bounded.

Since the hyperbolic spaces contain all normed linear spaces and their convex subsets, so uniformly convex Banach space is contained in hyperbolic metric space so it is natural to generalize the above result to hyperbolic metric space.

Definition 7 [16]. A bounded sequence $\left(\xi_{n}\right)$ in $H$ is known as $\Delta$-converge to an element $\xi \in H$, if $\xi$ is $a$ unique asymptotic centre of each subsequence $\left\{\xi_{n_{k}}\right\}$ of $\left\{\xi_{n}\right\}$.

In this section, following definitions and lemma are stated in [9].

Definition 8. Let $W$ be a nonempty set of a hyperbolic metric space $(H, \sigma)$. A map $\tau: W \longrightarrow[0, \infty)$ is said to be a type function, if there exists a bounded sequence $\left\{u_{n}\right\}$ in $H$ such that

$$
\tau(u)=\limsup _{n \longrightarrow \infty} \sigma\left(u_{n}, u\right) \text {, for all } u \in W .
$$

It is known that each bounded sequence generates a unique type function.

Lemma 9. Let $(H, \sigma, \preceq)$ be a uniformly convex hyperbolic metric space and $W$ a nonempty closed convex subset of $H$. Let $\tau: W \longrightarrow[0, \infty)$ be a type function. Then, $\tau$ is continuous. Furthermore, there exists a unique minimum point $\xi \in W$ 
such that

$$
\tau(\xi)=\inf \{\tau(u): u \in W\}
$$

Definition 10. A hyperbolic space $(H, \sigma)$ known as uniformly convex, if for every $r>0$ and $\varepsilon>0$,

$$
\begin{aligned}
\delta(r, \varepsilon) & =\inf \left\{1-\frac{1}{r} \sigma\left(\frac{1}{2} \xi \oplus \frac{1}{2} \eta, a\right) ; \sigma(\xi, a)\right. \\
& \leq r, \sigma(\eta, a) \leq r, \sigma(\xi, \eta) \geq r \varepsilon\}>0,
\end{aligned}
$$

for any $a \in \mathrm{H}$.

Definition 11. Let $W$ be a nonempty subset of a hyperbolic space $H$ and $\left\{\xi_{v}\right\}$ be a bounded sequence in $H$. Then, for every $\xi \in H$, define

(i) Asymptotic radius of $\left\{\xi_{v}\right\}$ at $\xi$ by

$$
r\left(\xi,\left\{\xi_{v}\right\}\right)=\underset{v \longrightarrow \infty}{\limsup } \sigma\left(\xi_{v}, \xi\right)
$$

(ii) Asymptotic radius of the sequence $\left\{\xi_{v}\right\}$ relative to the above supposed set $W$ by

$$
r\left(\xi,\left\{\xi_{v}\right\}\right)=\inf \left\{r\left(\xi, \xi_{v}\right): \xi \in W\right\}
$$

(iii) Asymptotic centre of the sequence $\left\{\xi_{v}\right\}$ relative to the above supposed set $W$ by

$$
A\left(W,\left\{\xi_{v}\right\}\right)=\left\{r\left(\xi,\left\{\xi_{v}\right\}\right)=r\left(W,\left\{\xi_{v}\right\}\right): \xi \in W\right\}
$$

Note that $A\left(W,\left\{\xi_{v}\right\}\right) \neq \varnothing$. Further, $A\left(W,\left\{\xi_{v}\right\}\right)$ has exactly one point if $H$ is uniformly convex.

From now to onward, we will suppose that the ordered intervals are convex and closed, and they are also contained in ordered hyperbolic space $(H, \preceq, F)$; these are described as follows:

$[a, \longrightarrow):=\{\xi \in H: a \leq \xi\}$ and $(\longleftarrow, b]:=\{\xi \in H: \xi \leq b\}$ for any $a, b \in H$.

\section{Monotone $\alpha$-Nonexpansive Mappings}

In this section, we will use the following iteration introduced by Kalsoom et al. [25].

$$
\left\{\begin{array}{l}
\xi \in W, \\
\xi_{v+1}=T\left(\left(1-\alpha_{v}\right) T \xi_{v}+\alpha_{v} T \eta_{v}\right), \\
\eta_{v}=\left(1-\beta_{v}\right) z_{v}+\beta_{v} T z_{v}, \\
z_{v}=\left(1-\gamma_{v}\right) \xi_{v}+\gamma_{v} T \xi_{v}, \quad v \in \mathbb{N}
\end{array}\right.
$$

where $\left\{\alpha_{v}\right\},\left\{\beta_{v}\right\}$ and $\left\{\gamma_{v}\right\}$ are in $(0,1)$.

Now we define monotone $\alpha$-nonexpansive mappings in partially ordered hyperbolic metric space $(H, \sigma, \preceq)$ as follows.

Definition 12. Let $W$ be a nonempty closed convex subset of an ordered hyperbolic metric space $H$. A self map $\mathscr{T}$ on $W$ is monotone $\alpha$-nonexpansive mapping, if $\mathscr{T}$ is monotone and for some $\alpha<1$,

$\sigma^{2}(\mathscr{T} \xi, \mathscr{T} \eta) \leq \alpha \sigma^{2}(\mathscr{T} \xi, \eta)+\alpha \sigma^{2}(\xi, \mathscr{T} \eta)+(1-2 \alpha) \sigma^{2}(\xi, \eta)$

for all $\xi, \eta \in W$ with $\xi \preceq \eta$.

Lemma 13. Let $W$ be a nonempty closed convex subset of an ordered hyperbolic metric space $H$. A self map $\mathscr{T}$ on $W$ is monotone $\alpha$-nonexpansive mapping; then,

(i) $\mathscr{T}$ is monotone quasi.

(ii) For all $\xi, \eta \in W$ with $\xi \preceq \eta$

$$
\begin{aligned}
\sigma^{2}(\mathscr{T} \xi, \mathscr{T} \eta) \leq & \sigma^{2}(\xi, \eta)+\frac{2 \alpha}{1-\alpha} \sigma^{2}(\mathscr{T} \xi, \xi) \\
& +\frac{2 \alpha}{1-\alpha} \sigma(\mathscr{T} \xi, \xi)[\sigma(\xi, \eta)+\sigma(\mathscr{T} \xi, \mathscr{T} \eta)] .
\end{aligned}
$$

Proof. To prove (i), it is followed by definition of monotone $\alpha$ -nonexpansive mappings that

$$
\sigma^{2}(\mathscr{T} \xi, \eta) \leq \alpha \sigma^{2}(\mathscr{T} \xi, \eta)+\alpha \sigma^{2}(\xi, \eta)+(1-2 \alpha) \sigma^{2}(\xi, \eta),
$$

implies $\sigma(\mathscr{T} \xi, \eta) \leq \sigma(\xi, \eta)$.

Hence, $\mathscr{T}$ is monotone quasi for $\xi \in W$ and $\eta \in F(\mathscr{T})$.

Now we will prove (ii), and if $0<\alpha<1$ then we have

$$
\begin{aligned}
\sigma^{2}(\mathscr{T} \xi, \mathscr{T} \eta) \leq & \alpha \sigma^{2}(\mathscr{T} \xi, \eta)+\alpha \sigma^{2}(\xi, \mathscr{T} \eta)+(1-2 \alpha) \sigma^{2}(\xi, \eta) \\
\leq & \alpha \sigma^{2}(\mathscr{T} \xi, \xi)+2 \alpha \sigma(\mathscr{T} \xi, \xi) \sigma(\xi, \eta)+\alpha \sigma^{2}(\xi, \eta) \\
& +\alpha \sigma^{2}(\mathscr{T} \xi, \mathscr{T} \eta)+2 \alpha \sigma(\mathscr{T} \xi, \mathscr{T} \eta) \sigma(\mathscr{T} \xi, \xi) \\
& +\alpha \sigma^{2}(\mathscr{T} \xi, \xi)+(1-2 \alpha) \sigma^{2}(\xi, \eta),
\end{aligned}
$$




$$
\begin{aligned}
\sigma^{2}(\mathscr{T} \xi, \mathscr{T} \eta) \leq & \sigma^{2}(\xi, \eta)+\frac{2 \alpha}{1-\alpha} \sigma^{2}(\mathscr{T} \xi, \xi) \\
& +\frac{2 \alpha}{1-\alpha} \sigma(\mathscr{T} \xi, \xi)[\sigma(\xi, \eta)+\sigma(\mathscr{T} \xi, \mathscr{T} \eta)]
\end{aligned}
$$

This completes the proof.

Definition 14. An ordered hyperbolic metric space $(H, \sigma, \preceq)$ is said to be uniformly convex, if for an arbitrary $h \in H, r>0$ and $\varepsilon>0$,

$$
\begin{aligned}
\delta(r, \varepsilon) & =\inf \left[1-\frac{1}{2} \sigma\left(\frac{1}{2} \xi \oplus \frac{1}{2} \eta, h\right) ; \sigma(\xi, h)\right. \\
& \leq r ; \sigma(\eta, h) \leq r ; \sigma(\xi, \eta) \geq r \varepsilon]>0 .
\end{aligned}
$$

Now, we utilize iteration processes for monotone $\alpha$ -nonexpansive mappings.

Lemma 15. Let $(H, \sigma, \preceq)$ be a uniformly convex partially ordered hyperbolic metric space (in short, UCPOHMS) and $W$ a nonempty closed convex subset of $H$. Let $\mathscr{T}: W \longrightarrow W$ be a monotone mapping and $\xi_{1} \in W$ be such that $\xi_{1} \preceq \mathscr{T} \xi_{1}[$ or $\left.T \xi_{1} \leq \xi_{1}\right]$. Then, for sequence $\left\{\xi_{v}\right\}$ defined by (14), we have

(a) $\xi_{v} \preceq \mathscr{T} \xi_{v} \preceq \xi_{v+1}\left(\right.$ or $\left.\xi_{v+1} \preceq \mathscr{T} \xi_{v} \preceq \xi_{v}\right)$;

(b) $\xi_{v} \preceq p\left(\operatorname{orp} \preceq \xi_{v}\right)$ provided $\left\{\xi_{v}\right\} \Delta$-converges to a point $p$ $\in W, \forall v \in \mathbb{N}$.

Theorem 16. Let $W$ be a nonempty closed convex subset of a $\operatorname{UCPOHMS}(H, \sigma, \preceq)$ and $\mathscr{T}: W \longrightarrow W$ be a monotone $\alpha$ -nonexpansive mapping. Assume that there exists $\xi_{1} \in W$ such that $\xi_{1} \preceq \mathscr{T} \xi_{1}$ and $\left\{\xi_{v}\right\}$ defined in (14) is a bounded sequence with $\xi_{v} \leq \eta$ for all $\eta \in W$ such that

$$
\liminf _{v \longrightarrow \infty} \sigma\left(\xi_{v}, \mathscr{T} \xi_{v}\right)=0
$$

Then, $F(\mathscr{T}) \neq \varnothing$.

Proof. Let $\left\{\xi_{v}\right\}$ defined by (14) be a bounded sequence such that

$$
\liminf _{v \longrightarrow \infty} \sigma\left(\xi_{v}, \mathcal{T} \xi_{v}\right)=0
$$

Then, there exists a subsequence $\left\{\xi_{v_{q}}\right\}$ such that

$$
\lim _{k \longrightarrow \infty} \sigma\left(\xi_{v_{q}}, \mathscr{T} \xi_{v_{q}}\right)=0
$$

By Lemma 15, we have

$$
\xi_{1} \preceq \xi_{v_{q}} \preceq \xi_{v_{q+1}}
$$

Define

$$
W_{q}=\left\{u \in W: \xi_{1} \preceq u\right\}
$$

for all $q \in \mathbb{N}$. Clearly, for every $q \in \mathbb{N}, W_{q}$ is closed convex. As $u \in W_{q}$, it shows that $W_{q} \neq \varnothing$. Define

$$
W_{\infty}=\bigcap_{q=1}^{\infty} W_{q} \neq \varnothing
$$
Then,

Then, $W_{\infty}$ is a closed convex subset of $W$. Let $u \in W_{\infty}$.

$$
\xi_{v_{q}} \preceq u, \forall q \in \mathbb{N}
$$

As we know, $\mathscr{T}$ is a mapping which is monotone; then, for $q \in \mathbb{N}$,

$$
\xi_{v_{q}} \preceq \mathscr{T} \xi_{v_{q}} \preceq \mathscr{T} u
$$

which implies that $\mathscr{T}\left(W_{\infty}\right) \subset W_{\infty}$. Let a type function $\tau$ $: W_{\infty} \longrightarrow[0, \infty)$ generated by $\left\{\xi_{v_{q}}\right\}$ such that

$$
\tau(u)=\underset{q \longrightarrow \infty}{\limsup } \sigma\left(\xi_{v_{q}}, u\right) .
$$

Then, there exists a unique point $z \in W_{\infty}$ such that

$$
\tau(z)=\inf \left\{\tau(u): u \in W_{\infty}\right\}
$$

By definition of type function,

$$
\tau(\mathscr{T} z)=\limsup _{q \longrightarrow \infty} \sigma\left(\xi_{v_{q}}, \mathscr{T} z\right)
$$

By using Lemma 13, we get

$$
\begin{aligned}
\sigma^{2}\left(\mathscr{T} \xi_{v_{q}}, \mathscr{T} \mathrm{z}\right) \leq & \sigma^{2}\left(\xi_{v_{q}}, z\right)+\frac{2 \alpha}{1-\alpha} \sigma^{2}\left(\mathscr{T} \xi_{v_{q}}, \xi_{v_{q}}\right) \\
& +\frac{2 \alpha}{1-\alpha} \sigma\left(\mathscr{T} \xi_{v_{q}}, \xi_{v_{q}}\right)\left[\sigma\left(\xi_{v_{q}}, z\right)\right. \\
& \left.+\sigma\left(\mathscr{T} \xi_{v_{q}}, \mathscr{T} \mathrm{z}\right)\right] .
\end{aligned}
$$

From the boundedness of the sequence $\left\{\xi_{v_{q}}\right\}$ and $\lim _{q \longrightarrow \infty} \sigma\left(\xi_{v_{q}}, \mathscr{T} \xi_{v_{q}}\right)=0$, we have

$$
\underset{q \longrightarrow \infty}{\lim \sup } \sigma^{2}\left(\mathscr{T} \xi_{v_{q}}, \mathscr{T} \mathrm{z}\right) \leq \underset{q \longrightarrow \infty}{\lim \sup } \sigma^{2}\left(\xi_{v_{q}}, z\right),
$$

implies $\tau(\mathrm{Tz})=\tau(z)$. It shows that $\mathscr{T} z=z$, and hence, $F(\mathscr{T}$ )$\neq \varnothing$.

Theorem 17. Let $W$ be a nonempty closed convex subset of a $\operatorname{UCPOHMS}(H, \sigma, \preceq)$ and $\mathscr{T}: W \longrightarrow W$ a monotone $\alpha$ 
-nonexpansive mapping. Assume that there exists $\xi_{1} \in W$ such that $\mathscr{T} \xi_{1} \preceq \xi_{1}$ and $\left\{\xi_{v}\right\}$ defined by (14) is a bounded sequence with $\xi_{v} \leq \eta$ for all $\eta \in W$ such that

$$
\liminf _{v \longrightarrow \infty} \sigma\left(\xi_{v}, \mathcal{T} \xi_{v}\right)=0
$$

Then, $F(\mathscr{T}) \neq \varnothing$.

Theorem 18. Let $(H, \sigma, \preceq)$ be a UCPOHMS, $C$ a closed convex cone, and $\mathscr{T}: C \longrightarrow C$ a monotone $\alpha$-nonexpansive mapping. Assume that $\xi_{1}=0$ and $\left\{\xi_{v}\right\}$ defined by (14) is a bounded sequence with $\xi_{v} \leq \eta$ for all $\eta \in W$ such that

$$
\liminf _{v \longrightarrow \infty} \sigma\left(\xi_{v}, \mathscr{T} \xi_{v}\right)=0
$$

Then, $F(\mathscr{T}) \neq \varnothing$.

Proof. With the help of definition of partial order $\preceq$, we know that $\xi_{1}=0 \preceq \mathscr{T} 0=\mathscr{T} \xi_{1}$; then, the proof is directly from Theorem 16 .

We now prove some convergence results for monotone $\alpha$ -nonexpansive mappings.

Lemma 19 [9]. Suppose $(H, \sigma)$ be a UC hyperbolic space and also monotone modulus of uniform convexity $\delta$, and suppose $z \in H$ and a sequence $\left\{\alpha_{v}\right\}$ such that $0<a \leq \alpha_{v} \leq b<1$. If the sequences $\left\{u_{v}\right\}$ and $\left\{v_{v}\right\}$ are in $H$, in such a way that $\lim \sup _{v \longrightarrow \infty} \sigma\left(u_{v}, z\right) \leq r, \quad \lim \sup _{v \rightarrow \infty} \sigma\left(v_{v}, z\right) \leq r, \quad$ and $\lim _{v \longrightarrow \infty} \sigma\left(\alpha_{v} u_{v} \oplus\left(1-\alpha_{v}\right) v_{v}, z\right)=r$, then $\lim _{v \longrightarrow \infty} \sigma\left(u_{v}, v_{v}\right)$ $=0$.

Theorem 20. Let $W$ be a nonempty closed convex subset of a $\operatorname{UCPOHMS}(H, \sigma, \preceq)$ and $\mathscr{T}: W \longrightarrow W$ a monotone $\alpha$-nonexpansive mapping. Suppose there exists a sequence $\left\{\xi_{v}\right\}$ defined by (14) with $\xi_{1} \preceq \mathscr{T} \xi_{1}\left(\right.$ or $\left.\mathscr{T} \xi_{1} \preceq \xi_{1}\right)$ and $F(\mathscr{T}) \neq \varnothing$. Then,

(1) $\left\{\xi_{v}\right\}$ is bounded

(2) $\sigma\left(\xi_{v+1}, z\right) \leq \sigma\left(\xi_{v}, z\right)$ and $\lim _{v \longrightarrow \infty} \sigma\left(\xi_{v}, z\right)$ exists for all $z \in F(\mathscr{T})$

(3) $\lim _{v \longrightarrow \infty} \sigma\left(\mathscr{T} \xi_{v}, \xi_{v}\right)=0$.

Proof. Suppose that $\xi_{1} \preceq z$ and $z \in F(\mathscr{T})$, and as we know that $\mathscr{T}$ is monotone, then $\mathscr{T} \xi_{1} \preceq \mathscr{T} z$, and so

$$
\xi_{1} \preceq \mathscr{T} \xi_{1} \preceq z
$$

It follows from Lemma 15.

$$
\xi_{v} \preceq \xi_{v+1} \preceq \mathscr{T} \xi_{v} \preceq z,
$$

which gives that

$$
\xi_{v+1} \preceq z, \forall v \in \mathbb{N} \text {. }
$$

It shows that $\left\{\xi_{v}\right\}$ is bounded. On the other hand, by using Lemma 13, we get

$$
\sigma\left(\xi_{v+1}, z\right) \leq \sigma\left(\xi_{v}, z\right)
$$

and so

$$
\begin{gathered}
\sigma\left(\xi_{v+1}, z\right) \leq \beta_{v} \sigma\left(\xi_{v}, z\right)+\left(1-\beta_{v}\right) \sigma\left(\mathscr{T} \xi_{v}, z\right) \\
\leq \beta_{v} \sigma\left(\xi_{v}, z\right)+\left(1-\beta_{v}\right) \sigma\left(\xi_{v}, z\right) \\
=\sigma\left(\xi_{v}, z\right) \\
\vdots \\
\leq \sigma\left(\xi_{1}, z\right) .
\end{gathered}
$$

Then, the sequence $\left\{\sigma\left(\xi_{v}, z\right)\right\}$ is nonincreasing and bounded sequence; hence, (i) and (ii) proved. So $\lim _{v \longrightarrow \infty} \sigma$ $\left(\xi_{v}, z\right)$ exists for all $z \in F(\mathscr{T})$ and $v \in \mathbb{N}$. Suppose that

$$
\lim _{v \longrightarrow \infty} \sigma\left(\xi_{v}, z\right) \leq s
$$

As $\mathscr{T}$ is monotone quasi,

$$
\limsup _{v \longrightarrow \infty} \sigma\left(\mathscr{T} \xi_{v}, z\right) \leq s
$$

and hence,

$$
\begin{gathered}
\limsup _{v \longrightarrow \infty} \sigma\left(\xi_{v+1}, z\right) \leq s, \\
s=\limsup _{v \longrightarrow \infty}\left\{\beta_{v} \sigma\left(\xi_{v}, z\right)+\left(1-\beta_{v}\right) \sigma\left(\mathscr{T} \xi_{v}, z\right)\right\} \\
=\limsup _{v \longrightarrow \infty} \sigma\left(\xi_{v}, z\right) .
\end{gathered}
$$

It concludes from Lemma 19 that

$$
\limsup _{v \longrightarrow \infty} \sigma\left(\xi_{v}, z\right)=0
$$

Theorem 21. Let $W$ be a nonempty closed convex subset of a $\operatorname{UCPOHMS}(H, \sigma, \preceq)$ and $\mathscr{T}: W \longrightarrow W$ a monotone $\alpha$-nonexpansive mapping. Suppose there exists a sequence $\left\{\xi_{v}\right\}$ defined by (14) with $\xi_{1} \preceq \mathscr{T} \xi_{1}\left(\right.$ or $\left.\mathscr{T} \xi_{1} \preceq \xi_{1}\right)$ and $F(\mathscr{T}) \neq \varnothing$. Then, $\left\{\xi_{v}\right\} \Delta$-converges to a fixed point of $\mathscr{T}$.

Proof. By the Theorem 18, we have $\left\{\xi_{v}\right\}$ is bounded. So, there exists a subsequence $\left\{\xi_{v_{q}}\right\}$ of $\left\{\xi_{v}\right\} \Delta$-converges to some $p \in$ $W$ such that

$$
\xi_{1} \preceq \xi_{v_{q}} \preceq p, \forall q \in \mathbb{N}
$$

In the next step, we prove there exists a unique $\Delta$-limit in $F(\mathscr{T})$ corresponding to each $\Delta$-convergent subsequence of $\left\{\xi_{v}\right\}$. Consider $\left\{\xi_{v}\right\}$ has two subsequences $\left\{\xi_{v_{q}}\right\}$ and $\left\{\xi_{v_{r}}\right\}$ which are $\Delta$-convergent to $l$ and $m$, respectively. Then, $\left\{\xi_{v}\right\}$ is bounded and 


$$
\lim _{q \longrightarrow \infty} \sigma\left(\mathscr{T} \xi_{v_{q}}, \xi_{v_{q}}\right)=0
$$

which concludes that $l \in F(\mathscr{T})$. Let $\tau: W \longrightarrow[0, \infty)$ is a type function which is generated by $\left\{\xi_{v_{q}}\right\}$. Then,

$$
\begin{gathered}
\tau(l)=\limsup _{v \longrightarrow \infty} \sigma\left(\xi_{v_{q}}, l\right), \\
\tau(\mathscr{T} l)=\limsup _{v \longrightarrow \infty} \sigma\left(\xi_{v_{q}}, \mathscr{T} l\right) .
\end{gathered}
$$

By Lemma 13, we infer

$$
\begin{aligned}
\sigma^{2}\left(\mathscr{T} \xi_{v_{q}}, \mathscr{T} l\right) \leq & \sigma^{2}\left(\xi_{v_{q}}, l\right)+\frac{2 \alpha}{1-\alpha} \sigma^{2}\left(\mathscr{T} \xi_{v_{q}}, \xi_{v_{q}}\right) \\
& +\frac{2 \alpha}{1-\alpha} \sigma\left(\mathscr{T} \xi_{v_{q}}, \xi_{v_{q}}\right)\left[\sigma\left(\xi_{v_{q}}, l\right)\right. \\
& \left.+\sigma\left(\mathscr{T} \xi_{v_{q}}, \mathscr{T} l\right)\right]
\end{aligned}
$$

as

$$
\begin{gathered}
\lim _{q \longrightarrow \infty} \sigma\left(\mathscr{T} \xi_{v_{q}}, \xi_{v_{q}}\right)=0 \\
\limsup _{q \longrightarrow \infty} \sigma\left(\mathscr{T} \xi_{v_{q}}, \mathscr{T} l\right) \leq \underset{q \longrightarrow \infty}{\lim \sup } \sigma\left(\xi_{v_{q}}, l\right) .
\end{gathered}
$$

By uniqueness of element $l$ and definition of $\Delta$-convergence, we conclude that

$$
\mathscr{T} l=l
$$

Similarly, one can easily show that

$$
\mathscr{T} m=m \text {. }
$$
get

By continuity of $\tau$ and definition of $\Delta$-convergence, we

$$
\begin{aligned}
\underset{v \longrightarrow \infty}{\limsup } \sigma\left(\xi_{v}, l\right) & \leq \underset{q \longrightarrow \infty}{\limsup } \sigma\left(\xi_{v_{q}}, l\right) \leq \underset{q \longrightarrow \infty}{\limsup } \sigma\left(\xi_{v_{q}}, m\right) \\
& =\underset{v \longrightarrow \infty}{\limsup } \sigma\left(\xi_{v}, m\right)=\underset{r \longrightarrow \infty}{\lim \sup } \sigma\left(\xi_{v_{r}}, m\right) \\
& \leq \underset{r \longrightarrow \infty}{\limsup } \sigma\left(\xi_{v_{r}}, l\right),
\end{aligned}
$$

which shows that $l=m$.

Theorem 22. Let $W$ be a nonempty closed convex subset of a $\operatorname{UCPOHMS}(H, \sigma, \preceq)$ and $\mathscr{T}: W \longrightarrow W$ a monotone $\alpha$-nonexpansive mapping. Suppose there exists a sequence $\left\{\xi_{v}\right\}$ defined by (14) with $\xi_{1} \preceq \mathscr{T} \xi_{1}\left(\right.$ or $\left.\mathscr{T} \xi_{1} \preceq \xi_{1}\right)$ and

$$
\limsup _{v \longrightarrow \infty} \beta_{v}\left(1-\beta_{v}\right)>0 \text {. }
$$

Then, $\left\{\xi_{v}\right\}$ converges strongly to a fixed point of $\mathscr{T}$.
Proof. By Theorem 18, there exists a subsequence $\left\{\xi_{v_{q}}\right\}$ of \{ $\left.\xi_{v}\right\}$ which converges strongly to a point $\eta \in W$. From Lemma 15 , we get

$$
\xi_{1} \preceq \xi_{v_{q}} \preceq \eta, \forall q \in \mathbb{N} \text {. }
$$

By Theorem 17, $F(\mathscr{T}) \neq \varnothing$ and $\left\{\xi_{v}\right\}$ is bounded, and

$$
\liminf _{v \longrightarrow \infty} \sigma\left(\mathscr{T} \xi_{v}, \xi_{v}\right)=0
$$

Without loss of generality, we get

$$
\lim _{q \longrightarrow \infty} \sigma\left(\mathscr{T} \xi_{v_{q}}, \xi_{v_{q}}\right)=0
$$

On the other hand, by Lemma 13, we derive

$$
\begin{aligned}
\sigma^{2}\left(\mathscr{T} \xi_{v_{q}}, \mathscr{T} \eta\right) \leq & \sigma^{2}\left(\xi_{v_{q}}, \eta\right)+\frac{2 \alpha}{1-\alpha} \sigma^{2}\left(\mathscr{T} \xi_{v_{q}}, \xi_{v_{q}}\right) \\
& +\frac{2 \alpha}{1-\alpha} \sigma\left(\mathscr{T} \xi_{v_{q}}, \xi_{v_{q}}\right)\left[\sigma\left(\xi_{v_{q}}, \eta\right)\right. \\
& \left.+\sigma\left(\mathscr{T} \xi_{v_{q}}, \mathscr{T} \eta\right)\right]
\end{aligned}
$$

as

$$
\begin{gathered}
\lim _{q \longrightarrow \infty} \sigma\left(\mathscr{T} \xi_{v_{q}}, \xi_{v_{q}}\right)=0, \\
\limsup _{q \longrightarrow \infty} \sigma\left(\mathscr{T} \xi_{v_{q}}, \mathscr{T} \eta\right) \leq \limsup _{q \longrightarrow \infty} \sigma\left(\xi_{v_{q}}, \eta\right) .
\end{gathered}
$$

By boundedness of $\left\{\xi_{v_{q}}\right\}$, we have

$$
\begin{gathered}
\limsup _{q \longrightarrow \infty} \sigma\left(\xi_{v_{q}}, \eta\right)=0, \\
\limsup _{q \longrightarrow \infty} \sigma\left(\mathscr{T} \xi_{v_{q}}, \mathscr{T} \eta\right)=0,
\end{gathered}
$$

and hence,

$$
\lim _{q \longrightarrow \infty} \sigma\left(\mathscr{T} \xi_{v_{q}}, \mathscr{T} \eta\right)=0
$$

Therefore,

$\lim _{q \longrightarrow \infty} \sigma\left(\xi_{v_{q}}, \mathscr{T} \eta\right)=\lim _{q \longrightarrow \infty} \sigma\left(\xi_{v_{q}}, \mathscr{T} \xi_{v_{q}}\right)+\lim _{q \longrightarrow \infty} \sigma\left(\mathscr{T} \xi_{v_{q}}, \mathscr{T} \eta\right)=0$

which shows that $\eta \in F(\mathscr{T})$. By Theorem $18, \lim _{v \longrightarrow \infty} \sigma\left(\xi_{v}\right.$, $\eta$ ) exists, so

$$
\lim _{v \longrightarrow \infty} \sigma\left(\xi_{v}, \eta\right)=0
$$

This completes the proof. 
Example 1. Let $W=[1,4]$ be a nonempty closed convex subset of a UCPOHMS $(\mathbb{R}, \sigma, \leq)$ and $T: W \longrightarrow W$ defined by $T x=(2 x+4)^{1 / 3}$ be a monotone $\alpha$-nonexpansive mapping. Then, for the sequences $\alpha_{n}=3 n /(2 n+1)^{4}, \beta_{n}=2 n+1 /$ $(7 n+3)^{2}$, and $\gamma_{n}=5 n+2 /(3 n+2)^{3}$, there exists a sequence $\left\{\xi_{v}\right\}$ for which all the conditions of Theorem 22 are satisfied by $T$, and hence, $x=2$ is the required fixed point.

\section{Comparison of Iteration Processes}

In this section, we are presenting some iterations [25-31] which we will be used in the numerical example.

Mann iteration process

In 1953, Mann proposed an iteration, namely, Mann iteration, for calculation of a fixed point for a nonexpansive mapping $\mathscr{T}$, defined as

$$
\xi_{v+1}=\beta_{v} \xi_{v}+\left(1-\beta_{v}\right) \mathscr{T} \xi_{v}
$$

for each $v \geq 1$ and $\left\{\beta_{v}\right\} \subset(0,1)$.

Ishikawa iteration process

In 1974, Ishikawa proposed the two-step iteration process as follows:

$$
\left\{\begin{array}{l}
\xi_{1}=\xi \in W, \\
\xi_{v+1}=\left(1-\alpha_{v}\right) \xi_{v}+\alpha_{v} \mathscr{T} \eta_{v}, \\
\eta_{v}=\left(1-\beta_{v}\right) \xi_{v}+\beta_{v} \mathscr{T} \xi_{v}, \quad v \in \mathbb{N},
\end{array}\right.
$$

where $\left\{\alpha_{v}\right\}$ and $\left\{\beta_{v}\right\}$ are in $(0,1)$.

Noor iteration process lows:

In 2000, Noor proposed the three-step iteration as fol-

$$
\left\{\begin{array}{l}
\xi_{1}=\xi \in W, \\
\xi_{v+1}=\left(1-\alpha_{v}\right) \xi_{v}+\alpha_{v} \mathscr{T} \eta_{v}, \\
\eta_{v}=\left(1-\beta_{v}\right) \xi_{v}+\beta_{v} \mathscr{T} \mathbf{z}_{v}, \\
z_{v}=\left(1-\gamma_{v}\right) \xi_{v}+\gamma_{v} \mathscr{T} \xi_{v}, \quad v \in \mathbb{N},
\end{array}\right.
$$

where $\left\{\alpha_{v}\right\},\left\{\beta_{v}\right\}$, and $\left\{\gamma_{v}\right\}$ are in $(0,1)$.

Agarwal iteration process

In 2007, Agarwal et al. introduced the three-step iteration as follows:

$$
\left\{\begin{array}{l}
\xi_{1}=\xi \in W, \\
\xi_{v+1}=\left(1-\alpha_{v}\right) \mathscr{T} \xi_{v}+\alpha_{v} \mathscr{T} \eta_{v}, \\
\eta_{v}=\left(1-\beta_{v}\right) \xi_{v}+\beta_{v} \mathscr{T} \xi_{v}, \quad v \in \mathbb{N},
\end{array}\right.
$$

where $\left\{\alpha_{v}\right\}$ and $\left\{\beta_{v}\right\}$ are in $(0,1)$.

Abbas and Nazir iteration process
TABle 1: Convergence behavior of Mann, Ishikawa, Noor, Agarwal, Abbas, Thakur, and Kalsoom et al. iterations towards fixed point.

\begin{tabular}{lcccc}
\hline Initial points & 0.5 & 0.7 & 1.3 & 1.8 \\
\hline Mann & 3978 & 3980 & 3981 & 3981 \\
Ishikawa & 2656 & 2657 & 2659 & 2600 \\
Noor & 2279 & 2280 & 2282 & 2283 \\
Agarwal & 1590 & 1590 & 1590 & 1576 \\
Abbas & 1000 & 1000 & 1000 & 1001 \\
Thakur & 883 & 883 & 883 & 882 \\
Kalsoom & 795 & 795 & 795 & 786 \\
\hline
\end{tabular}

In 2014, Abbas and Nazir introduced the three-step iteration as follows:

$$
\left\{\begin{array}{l}
\xi_{1}=\xi \in W, \\
\xi_{v+1}=\left(1-\alpha_{v}\right) \mathscr{T} \mathrm{z}_{v}+\alpha_{v} \mathscr{T} \eta_{v}, \\
\eta_{v}=\left(1-\beta_{v}\right) \mathscr{T} \xi_{v}+\beta_{v} \mathscr{T} \mathrm{z}_{v}, \\
z_{v}=\left(1-\gamma_{v}\right) \xi_{v}+\gamma_{v} \mathscr{T} \xi_{v}, \quad v \in \mathbb{N},
\end{array}\right.
$$

where $\left\{\alpha_{v}\right\},\left\{\beta_{v}\right\}$, and $\left\{\gamma_{v}\right\}$ are in $(0,1)$.

Thakur iteration process

In 2016, Thakur et al. proposed the three-step iteration as follows:

$$
\left\{\begin{array}{l}
\xi_{1}=\xi \in W, \\
\xi_{v+1}=\mathscr{T} \eta_{v}, \\
\eta_{v}=\mathscr{T}\left(\left(1-\alpha_{v}\right) \xi_{v}+\alpha_{v} z_{v}\right), \\
z_{v}=\left(1-\beta_{v}\right) \xi_{v}+\beta_{v} \mathscr{T} \xi_{v}, \quad v \in \mathbb{N},
\end{array}\right.
$$

where $\left\{\alpha_{v}\right\}$ and $\left\{\beta_{v}\right\}$ are in $(0,1)$.

Two qualities fastness and stability play a vital role in iteration process to be performed. In [32], Rhoades mentioned that for the increasing functions, Ishikawa [27] iteration process is faster than Mann iteration process [26] but in the case of decreasing function, condition is reverse. In [29], Agarwal et al. proved that their iteration process was more stable than the previous ones. In [31], Thakur iteration process was considered faster convergent than all the abovementioned iteration processes.

Recently, Kalsoom et al. [25] introduced a new iteration process and proved it to be the fastest convergent than all. The following example is given to support this claim.

Example 2. Define

$$
\mathscr{T} \xi=\sin \xi-1+\cos \xi
$$

for $\xi \in[0,2)$. Then, $\mathscr{T}$ is monotone nonexpansive mapping as well as $\mathscr{T}$ is monotone $\alpha$-nonexpansive mapping. 


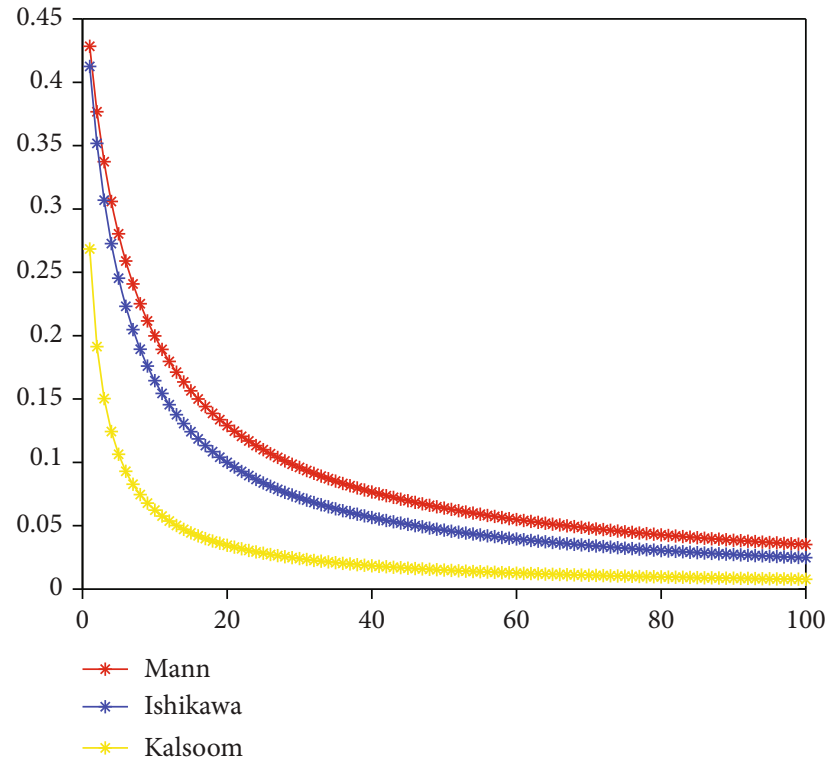

FIGURE 1: Convergence behavior of Mann, Ishikawa, and Kalsoom et al. iterations towards fixed point.

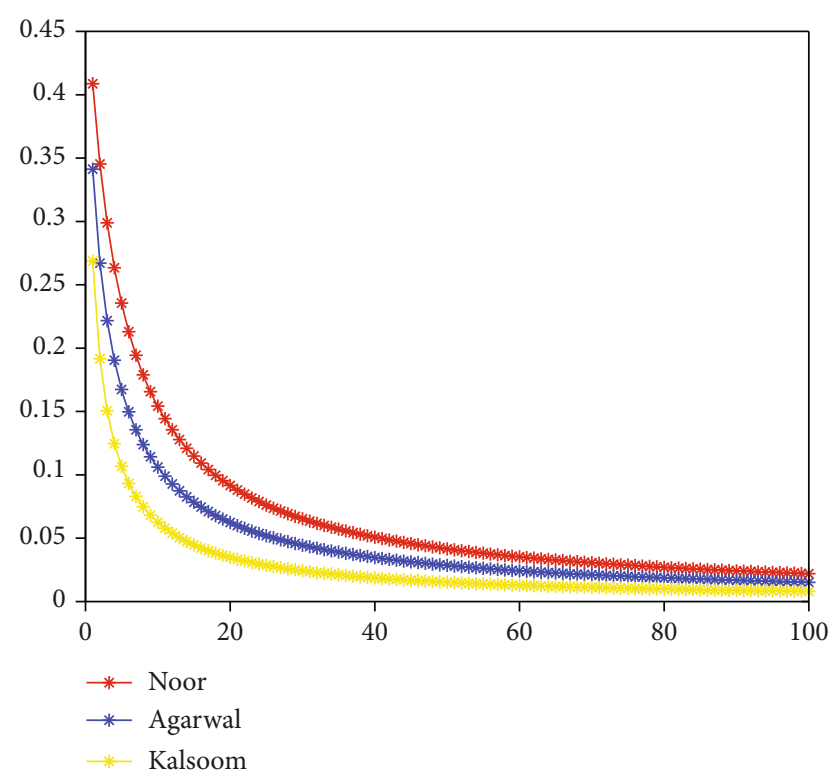

Figure 2: Convergence behavior of Noor, Agarwal, and Kalsoom et al. iterations towards fixed point.

Now we will compare abovementioned iteration processes to check the convergence of mapping $\mathscr{T}$. By using MATLAB, we present graphs and table.

In Table 1, we discussed the convergence behavior of some iteration processes. It is clear that all iterations approach to 0 which is the fixed point of mapping $\mathscr{T}$. In this case, Figures 1-3 show that Kalsoom et al. iteration process converges faster to the fixed point as compared the other iterations.

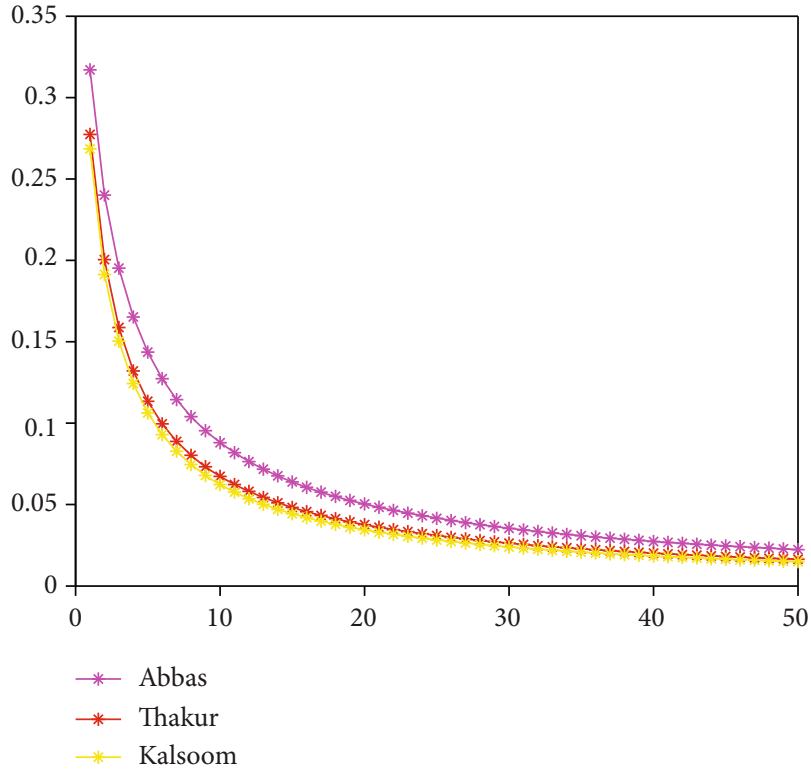

Figure 3: Convergence behavior of Abbas, Thakur, and Kalsoom et al. iterations towards fixed point.

\section{Monotone Generalized $\beta$-Nonexpansive Mappings}

In this section, we define monotone generalized $\beta$-nonexpansive mapping which generalizes the results of Pandey and Shukla [13] in hyperbolic spaces.

Now we will define monotone generalized $\beta$-nonexpansive mappings in hyperbolic space with nontrivial example.

Definition 23. Let $W$ be a nonempty subset of an ordered hyperbolic metric space $(H, \sigma, \preceq)$. A mapping $\mathscr{T}: W \longrightarrow$ $W$ is said to be monotone generalized $\beta$-nonexpansive mapping, if $\mathscr{T}$ is monotone and there exists $\beta \in[0,1)$ such that

$$
\begin{aligned}
\frac{1}{2} \sigma(\xi, \mathscr{T} \xi) & \leq \sigma(\xi, \eta) \Rightarrow \sigma(\mathscr{T} \xi, \mathscr{T} \eta) \\
& \leq \sigma(\xi, \eta)+\beta\{\sigma(\xi, \eta)-\sigma(\mathscr{T} \xi, \mathscr{T} \eta)\}
\end{aligned}
$$

for all $\xi, \eta \in W$ with $\xi \preceq \eta$.

Proposition 24. Every monotone nonexpansive mapping is monotone generalized $\beta$-nonexpansive mapping, but converse is not true.

Proof. By putting $\beta=0$ in (69), we have

$$
\frac{1}{2} \sigma(\xi, \mathscr{T} \xi) \leq \sigma(\xi, \eta) \Rightarrow \sigma(\mathscr{T} \xi, \mathscr{T} \eta) \leq \sigma(\xi, \eta)
$$

which shows that monotone generalized $\beta$-nonexpansive mapping reduces to monotone nonexpansive mapping satisfying the condition (3). The following example will prove the converse statement does not hold. 
Example 3. Let $W=[0,4]$ be a subset of $H$ endowed with usual order. Define $\mathscr{T}: W \longrightarrow W$ by

$$
\mathscr{T} \xi= \begin{cases}0, & \xi \neq 4, \\ 2, & \xi=4 .\end{cases}
$$

Then, for $\xi \in(2,8 / 3]$ and $\eta=4$,

$$
\frac{1}{2} \sigma(\xi, \mathscr{T} \xi) \leq \sigma(\xi, \eta)
$$

implies

$$
\sigma(\mathscr{T} \xi, \mathscr{T} \eta)=2>\sigma(\xi, \eta)
$$

and so $\mathscr{T}$ does not hold condition (3). Again, $\xi \in(2,3]$ and $\eta=4$

$$
\frac{1}{2} \sigma(\eta, \mathscr{T} \eta) \leq \sigma(\xi, \eta) \Rightarrow \sigma(\mathscr{T} \xi, \mathscr{T} \eta)>\sigma(\xi, \eta)
$$

and $\mathscr{T}$ does not satisfy condition (3). Nevertheless, $\mathscr{T}$ is generalized $\beta$-nonexpansive mapping with $\beta \geq 1 / 3$.

Proposition 25. Let $W$ be a nonempty subset of an ordered hyperbolic metric space $(H, \sigma, \preceq)$ and $\mathscr{T}: W \longrightarrow W$ a monotone generalized $\beta$-nonexpansive mapping which has a fixed point $\eta \in W$ with $\xi \preceq \eta$. Then, $\mathscr{T}$ can be referred as a monotone quasi-nonexpansive mapping.

Proof. Let $\eta \in F(\mathscr{T})$ and $\xi \in W$; then, by (69),

$$
\begin{aligned}
& \frac{1}{2} \sigma(\xi, \mathscr{T} \xi) \leq \sigma(\xi, \eta) \Rightarrow \sigma(\mathscr{T} \xi, \mathscr{T} \eta) \\
& \leq \sigma(\xi, \eta)+\beta\{\sigma(\xi, \eta)-\sigma(\mathscr{T} \xi, \mathscr{T} \eta)\}, \\
& \sigma(\mathscr{T} \xi, \eta) \leq \sigma(\xi, \eta)+\beta \sigma(\xi, \eta)-\beta \sigma(\mathscr{T} \xi, \eta), \\
&(1+\beta) \sigma(\mathscr{T} \xi, \eta) \leq(1+\beta) \sigma(\xi, \eta),
\end{aligned}
$$

where $(1+\beta)>0$ as $\beta \in[0,1)$ which shows that $\mathscr{T}$ is monotone quasi-nonexpansive mapping.

Proposition 26. Let $W$ be a nonempty subset of an ordered hyperbolic metric space $(H, \sigma, \preceq)$. If $\mathscr{T}: W \longrightarrow W$ is monotone generalized $\beta$-nonexpansive mapping, then $F(\mathscr{T})$ is closed. Furthermore, if $H$ is strictly convex, then $W$ is convex and $F(\mathscr{T})$ is also convex.

Proof. Let $\left\{z_{v}\right\}$ be a sequence in $F(\mathscr{T})$ which converges to $z$ $\in W$. Since

$$
\frac{1}{2} \sigma\left(z_{v}, \mathscr{T} z_{v}\right) \leq \sigma\left(z_{v}, z\right)
$$

with the help of continuity of metric, we have

$$
\begin{gathered}
\lim _{v \longrightarrow \infty} \sigma\left(\mathscr{T}_{\mathrm{z}_{v}}, \mathscr{T} \mathrm{z}\right) \leq \lim _{v \longrightarrow \infty} \sigma\left(z_{v}, \mathscr{T} \mathrm{z}\right), \\
\lim _{v \longrightarrow \infty} \sigma\left(z_{v}, \mathscr{T} \mathrm{z}\right) \leq \lim _{v \longrightarrow \infty}\left[\sigma\left(z_{v}, z\right)+\beta\left\{\sigma\left(z_{v}, z\right)-\sigma\left(\mathscr{T}_{v}, \mathscr{T} \mathrm{z}\right)\right\}\right], \\
\lim _{v \longrightarrow \infty} \sigma\left(z_{v}, \mathscr{T} \mathrm{z}\right) \leq \lim _{v \longrightarrow \infty} \sigma\left(z_{v}, z\right)+\beta \lim _{v \longrightarrow \infty} \sigma\left(z_{v}, z\right)-\beta \lim _{v \longrightarrow \infty} \sigma\left(z_{v}, \mathscr{T} \mathrm{z}\right), \\
(1+\beta) \lim _{v \longrightarrow \infty} \sigma\left(z_{v}, \mathscr{T} \mathrm{z}\right) \leq(1+\beta) \lim _{v \longrightarrow \infty} \sigma\left(z_{v}, z\right), \\
(1+\beta)>0 \quad \text { as } \beta \in[0,1), \\
\lim _{v \longrightarrow \infty} \sigma\left(z_{v}, \mathscr{T} \mathrm{z}\right) \leq \lim _{v \longrightarrow \infty} \sigma\left(z_{v}, z\right), \mathscr{T} \mathrm{z}=z .
\end{gathered}
$$

Hence, $F(\mathscr{T})$ is closed. Now, we assume that $H$ is strictly convex and $W$ is convex. Let $\lambda \in[0,1), \xi, \eta \in F(\mathscr{T})$ with $\xi \neq \eta$ ; then, put $z=\lambda \xi \oplus(1-\lambda) \eta \in W$.

$$
\begin{gathered}
\frac{1}{2} \sigma(\xi, \mathscr{T} \xi)=0<\sigma(\xi, z), \\
\sigma(\mathscr{T} \xi, \mathscr{T} \mathrm{z}) \leq \sigma(\xi, z)+\beta\{\sigma(\xi, z)-\sigma(\mathscr{T} \xi, \mathscr{T} \mathrm{z})\} \\
(1+\beta) \sigma(\mathscr{T} \xi, \mathscr{T} \mathrm{z}) \leq(1+\beta) \sigma(\xi, z), \\
(1+\beta)>0 \text { as } \beta \in[0,1), \\
\Rightarrow \sigma(\mathscr{T} \xi, \mathscr{T} z) \leq \sigma(\xi, z) .
\end{gathered}
$$

By similar argument, we get

$$
\begin{gathered}
\frac{1}{2} \sigma(\eta, \mathscr{T} \eta)=0<\sigma(\eta, z) \\
\sigma(\mathscr{T} \eta, \mathscr{T} \mathrm{z}) \leq \sigma(\eta, z)+\beta\{\sigma(\eta, z)-\sigma(\mathscr{T} \eta, \mathscr{T} \mathrm{z})\} \\
(1+\beta) \sigma(\mathscr{T} \eta, \mathscr{T} \mathrm{z}) \leq(1+\beta) \sigma(\eta, z) \\
(1+\beta)>0 \text { as } \beta \in[0,1) \\
\Rightarrow \sigma(\mathscr{T} \eta, \mathscr{T} z) \leq \sigma(\eta, z) .
\end{gathered}
$$

Therefore,

$$
\begin{aligned}
\sigma(\xi, \eta) & \leq \sigma(\xi, \mathscr{T} \mathrm{z})+\sigma(\mathscr{T} \mathrm{z}, \eta) \\
& =\sigma(\mathscr{T} \xi, \mathscr{T} \mathrm{z})+\sigma(\mathscr{T} \mathrm{z}, \mathscr{T} \eta) \\
& \leq \sigma(\xi, z)+\sigma(z, \eta) \leq \sigma(\xi, \eta) .
\end{aligned}
$$

From strict convexity of $H$, there exists $\mu \in[0,1)$ such that

$$
\begin{gathered}
\mathscr{T} \mathrm{Z}=\mu \xi \oplus(1-\mu), \\
\sigma(\xi, \mathscr{T} \xi) \leq \sigma(\xi, z), \\
\sigma(\xi, \mu \xi \oplus(1-\mu) \eta) \leq \sigma(\xi, z), \\
(1-\mu) \sigma(\xi, \eta) \leq \sigma(\xi, z) \eta .
\end{gathered}
$$

By using value of $\xi$, it gives

$$
(1-\mu) \sigma(\xi, \eta) \leq(1-\lambda) \sigma(\xi, \eta)
$$


and similarly,

$$
\sigma(\eta, \mathscr{T} \mathrm{z}) \leq \sigma(\eta, z) .
$$

By putting values of $z$ and $\mathscr{T} z$, we infer

$$
\mu \sigma(\xi, \eta) \leq \lambda \sigma(\xi, \eta)
$$

From the above two inequalities, it is concluded that

$$
\begin{aligned}
(1-\mu) & \leq(1-\lambda), \mu \leq \lambda, \\
\mu & =\lambda, \\
z & =\mathscr{T}_{\mathrm{z}} \Rightarrow z \in F(\mathscr{T}) .
\end{aligned}
$$

Hence, $F(\mathscr{T})$ is convex.

Lemma 27. Let $W$ be a nonempty subset of an ordered hyperbolic metric space $(H, \sigma, \preceq)$ and $\mathscr{T}: W \longrightarrow W$ a monotone generalized $\beta$-nonexpansive mapping. Then, for each $\xi, \eta \in$ $W$ with $\xi \leq \eta$,

(a) $\sigma\left(T \xi, T^{2} \xi\right) \leq \sigma(\xi, T \xi)$.

(b) Either $1 / 2 \sigma(\xi, T \xi) \leq \sigma(\xi, \eta)$ or $1 / 2 \sigma\left(T \xi, T^{2} \xi\right) \leq \sigma(T \xi$ $\eta)$.

(c) Either $\sigma(T \xi, T \eta) \leq \sigma(\xi, \eta)+\beta\{\sigma(\xi, \eta) \sigma(T \xi, T \eta)\}$ or $\sigma\left(T^{2} \xi, T^{2} \eta\right) \leq \sigma(T \xi, \eta)+\beta\left\{-\sigma(T \xi, \eta)-\sigma\left(T^{2} \xi, T^{2} \eta\right)\right.$ \} .

Proof. Since

$$
\frac{1}{2} \sigma(\xi, \mathscr{T} \xi) \leq \sigma(\xi, \mathscr{T} \xi)
$$

implies

$$
\begin{gathered}
\sigma\left(\mathscr{T} \xi, \mathscr{T}^{2} \xi\right) \leq \sigma(\xi, \mathscr{T} \xi)+\beta\left\{\sigma(\xi, \mathscr{T} \xi)-\sigma\left(\mathscr{T} \xi, \mathscr{T}^{2} \xi\right)\right\}, \\
(1+\beta) \sigma\left(\mathscr{T} \xi, \mathscr{T}^{2} \xi\right) \leq(1+\beta) \sigma(\xi, \mathscr{T} \xi), \\
(1+\beta)>0, \beta \in[0,1), \\
\sigma\left(\mathscr{T} \xi, \mathscr{T}^{2} \xi\right) \leq \sigma(\xi, \mathscr{T} \xi) .
\end{gathered}
$$

Hence, part (a) is satisfied. Now, we will prove part (b); we argue with contradiction, and suppose

$$
\begin{gathered}
\frac{1}{2} \sigma(\xi, \mathscr{T} \xi)>\sigma(\xi, \eta), \\
\frac{1}{2} \sigma\left(\mathscr{T} \xi, \mathscr{T}^{2} \xi\right)>\sigma(\mathscr{T} \xi, \eta) .
\end{gathered}
$$

By (a) and triangular inequality,

$$
\begin{aligned}
\sigma(\xi, \mathscr{T} \xi) & \leq \sigma(\xi, \eta)+\sigma(\mathscr{T} \xi, \eta)<\frac{1}{2} \sigma(\xi, \mathscr{T} \xi)+\frac{1}{2} \sigma\left(\mathscr{T} \xi, \mathscr{T}^{2} \xi\right) \\
& \leq \frac{1}{2} \sigma(\xi, \mathscr{T} \xi)+\frac{1}{2} \sigma(\xi, \mathscr{T} \xi) \leq \sigma(\xi, \mathscr{T} \xi),
\end{aligned}
$$

which is contradiction to our supposition, hence proved. The proof of (c) is in a similar way, so we omit that.

Lemma 28. Let $W$ be a nonempty subset of an ordered hyperbolic metric space $(H, \sigma, \leq)$ and $\mathscr{T}: W \longrightarrow W$ a monotone generalized $\beta$-nonexpansive mapping. Then, for each $\xi, \eta \in$ $W$ with $\xi \preceq \eta$,

$$
\sigma(\xi, \mathscr{T} \eta) \leq\left(\frac{2+\beta}{1-\beta}\right) \sigma(\xi, \mathscr{T} \xi)+\left(\frac{1+\beta}{1-\beta}\right) \sigma(\xi, \eta) .
$$

Proof. By the help of Lemma 27, we infer either

$$
\sigma(\mathscr{T} \xi, \mathscr{T} \eta) \leq \sigma(\xi, \eta)+\beta\{\sigma(\xi, \eta)-\sigma(\mathscr{T} \xi, \mathscr{T} \eta)\}
$$

or

$$
\sigma\left(\mathscr{T}^{2} \xi, \mathscr{T}^{2} \eta\right) \leq \sigma(\mathscr{T} \xi, \eta)+\beta\left\{\sigma(\mathscr{T} \xi, \eta)-\sigma\left(\mathscr{T}^{2} \xi, \mathscr{T}^{2} \eta\right)\right\}
$$

In the first case, we have

$$
\begin{aligned}
\sigma(\xi, \mathscr{T} \eta) & \leq \sigma(\xi, \mathscr{T} \xi)+\sigma(\xi, \eta)+\beta\{\sigma(\xi, \eta)-\sigma(\mathscr{T} \xi, \mathscr{T} \eta)\} \\
& \leq \sigma(\xi, \mathscr{T} \xi)+\sigma(\xi, \eta)+\beta\{\sigma(\xi, \mathscr{T} \xi)+\sigma(\mathscr{T} \xi, \eta)-\sigma(\mathscr{T} \xi, \mathscr{T} \eta)\} \\
& \leq \sigma(\xi, \mathscr{T} \xi)+\sigma(\xi, \eta)+\beta\{\sigma(\xi, \mathscr{T} \xi)+\sigma(\xi, \eta)-\sigma(\xi, \mathscr{T} \eta)\} \\
& \leq \sigma(\xi, \mathscr{T} \xi)+\sigma(\xi, \eta)+\beta\{\sigma(\xi, \mathscr{T} \xi)+\sigma(\eta, \mathscr{T} \eta)\} \\
& \leq \sigma(\xi, \mathscr{T} \xi)+\sigma(\xi, \eta)+\beta \sigma(\xi, \mathscr{T} \xi)+\beta \sigma(\xi, \eta)+\beta \sigma(\xi, \mathscr{T} \eta),
\end{aligned}
$$

and so

$$
\sigma(\xi, \mathscr{T} \eta) \leq\left(\frac{1+\beta}{1-\beta}\right) \sigma(\xi, \mathscr{T} \xi)+\left(\frac{1+\beta}{1-\beta}\right) \sigma(\xi, \eta) .
$$

In second case,

$$
\begin{aligned}
\sigma(\xi, \mathscr{T} \eta) & \leq \sigma(\xi, \mathscr{T} \xi)+\sigma\left(\mathscr{T} \xi, \mathscr{T}^{2} \xi\right)+\sigma\left(\mathscr{T}^{2} \xi, \mathscr{T} \eta\right) \\
& \leq \sigma(\xi, \mathscr{T} \xi)+\sigma(\xi, \mathscr{T} \xi)+\sigma\left(\mathscr{T}^{2} \xi, \mathscr{T} \eta\right) \\
& \leq 2 \sigma(\xi, \mathscr{T} \xi)+\sigma(\mathscr{T} \xi, \eta)+\beta\left\{\sigma(\mathscr{T} \xi, \eta)+\sigma\left(\mathscr{T}^{2} \xi, \mathscr{T} \eta\right)\right\} \\
& \leq 2 \sigma(\xi, \mathscr{T} \xi)+\sigma(\mathscr{T} \xi, \eta)+\beta\{\sigma(\xi, \mathscr{T} \xi)+\sigma(\eta, \mathscr{T} \eta)\} \\
& \leq 2 \sigma(\xi, \mathscr{T} \xi)+\sigma(\xi, \eta)+\beta \sigma(\xi, \mathscr{T} \xi)+\beta \sigma(\xi, \eta)+\beta \sigma(\xi, \mathscr{T} \eta)
\end{aligned}
$$


TABle 2: Convergence behavior of Mann, Ishikawa, Noor, Agarwal, Abbas, Thakur and Kalsoom et al. iterations towards fixed point.

\begin{tabular}{|c|c|c|c|c|c|c|c|}
\hline Steps & Mann & Ishikawa & Noor & Agarwal & Abbas & Thakur & Kalsoom \\
\hline 1 & 4.807669 & 4.457118 & 4.440406 & 4.264788 & 4.183261 & 4.098255 & 4.080140 \\
\hline 2 & 4.653029 & 4.210612 & 4.195196 & 4.072351 & 4.034941 & 4.010133 & 4.006740 \\
\hline 3 & 4.528465 & 4.097407 & 4.086770 & 4.019942 & 4.006713 & 4.001050 & 4.000569 \\
\hline 4 & 4.427974 & 4.045132 & 4.038623 & 4.005510 & 4.001291 & 4.000108 & 4.000048 \\
\hline 5 & 4.346800 & 4.020928 & 4.017202 & 4.001523 & 4.000248 & 4.000011 & 4.000000 \\
\hline 6 & 4.281160 & 4.009708 & 4.007663 & 4.000421 & 4.000047 & 4.000001 & 4.000000 \\
\hline 7 & 4.228036 & 4.004504 & 4.003414 & 4.000116 & 4.000009 & 4.000000 & 4.000000 \\
\hline 8 & 4.185011 & 4.002090 & 4.001521 & 4.000032 & 4.000001 & 4.000000 & 4 \\
\hline 9 & 4.150144 & 4.000970 & 4.000677 & 4.000008 & 4.000000 & 4.000000 & 4 \\
\hline 10 & 4.121875 & 4.000450 & 4.000302 & 4.000002 & 4.000000 & 4.000000 & 4 \\
\hline 11 & 4.098946 & 4.000208 & 4.000134 & 4.000000 & 4.000000 & 4.000000 & 4 \\
\hline 12 & 4.080342 & 4.000096 & 4.000059 & 4.000000 & 4.000000 & 4 & 4 \\
\hline 13 & 4.065244 & 4.000044 & 4.000026 & 4.000000 & 4 & 4 & 4 \\
\hline 14 & 4.052988 & 4.000020 & 4.000011 & 4.000000 & 4 & 4 & 4 \\
\hline 15 & 4.043038 & 4.000009 & 4.000005 & 4.000000 & 4 & 4 & 4 \\
\hline 16 & 4.034959 & 4.000004 & 4.000002 & 4.000000 & 4 & 4 & 4 \\
\hline 17 & 4.028397 & 4.000002 & 4.000001 & 4 & 4 & 4 & 4 \\
\hline 18 & 4.023069 & 4.000000 & 4.000000 & 4 & 4 & 4 & 4 \\
\hline 19 & 4.018740 & 4.000000 & 4.000000 & 4 & 4 & 4 & 4 \\
\hline 20 & 4.015225 & 4.000000 & 4.000000 & 4 & 4 & 4 & 4 \\
\hline 21 & 4.012369 & 4 & 4 & 4 & 4 & 4 & 4 \\
\hline 22 & 4.010049 & 4 & 4 & 4 & 4 & 4 & 4 \\
\hline 23 & 4.008164 & 4 & 4 & 4 & 4 & 4 & 4 \\
\hline 24 & 4.006633 & 4 & 4 & 4 & 4 & 4 & 4 \\
\hline 25 & 4.006633 & 4 & 4 & 4 & 4 & 4 & 4 \\
\hline
\end{tabular}

and hence,

$$
\sigma(\xi, \mathscr{T} \eta) \leq\left(\frac{2+\beta}{1-\beta}\right) \sigma(\xi, \mathscr{T} \xi)+\left(\frac{1+\beta}{1-\beta}\right) \sigma(\xi, \eta)
$$

Hence, we get the desired result.

Theorem 29. Let $W$ be a nonempty convex and closed subset of an ordered hyperbolic metric space $(H, \sigma, \preceq)$ and $\mathscr{T}: W$ $\longrightarrow W$ a monotone generalized $\beta$-nonexpansive mapping. Then, $F(\mathscr{T}) \neq \varnothing$ iff $\left\{T^{v} \xi\right\}$ is a sequence which is also bounded for some $\xi \in W$ provides that $\mathscr{T}^{v} \xi \preceq z$ for some $z \in W$ and $\xi \preceq$ $\mathscr{T} \xi$.

Proof. Let $\left\{\mathscr{T}^{v} \xi\right\}$ be a bounded sequence for some $\xi \in W$. As we know that $\mathscr{T}$ is monotone and $\xi \preceq \mathscr{T} \xi$, so we get

$$
\mathscr{T} \xi \preceq \mathscr{T}^{2} \xi
$$

In the same manner, we get

$$
\mathscr{T}^{2} \xi \preceq \mathscr{T}^{3} \xi \preceq \mathscr{T}^{4} \xi \preceq \cdots \preceq \mathscr{T}^{v} \xi \preceq \mathscr{T}^{v+1} \xi \preceq \cdots
$$

Define $\xi_{v}=\mathscr{T} \xi_{v-1}=\mathscr{T}^{v} \xi, \forall v \in \mathbb{N}$. Then, the asymptotic centre of $\left\{\xi_{v}\right\}$ w.r.t $W$ is $A\left(W,\left\{\xi_{v}\right\}\right)=\{z\}$ where $z$ is unique and $\xi_{v} \preceq z$ for all $v \in \mathbb{N}$. Now, we claim that $\left\{\sigma\left(\xi_{v+1}, \xi_{v+2}\right)\right\}$ is a nonincreasing sequence, that is,

$$
\sigma\left(\xi_{v+1}, \xi_{v+2}\right) \leq \sigma\left(\xi_{v}, \xi_{v+1}\right)
$$

Since

$$
\frac{1}{2} \sigma\left(\xi_{v}, \mathscr{T} \xi_{v}\right) \leq \sigma\left(\xi_{v}, \xi_{v+1}\right)
$$

which gives that

$$
\begin{aligned}
\sigma\left(\xi_{v+1}, \xi_{v+2}\right) & =\sigma\left(\mathscr{T} \xi_{v}, \mathscr{T} \xi_{v+1}\right) \\
& \leq \sigma\left(\xi_{v}, \xi_{v+1}\right)+\beta\left\{\sigma\left(\xi_{v}, \xi_{v+1}\right)-\sigma\left(\mathscr{T} \xi_{v}, \mathscr{T} \xi_{v+1}\right)\right\} \\
& =\sigma\left(\xi_{v}, \xi_{v+1}\right)+\beta\left\{\sigma\left(\xi_{v}, \xi_{v+1}\right)-\sigma\left(\xi_{v+1}, \xi_{v+2}\right)\right\} \\
& \Longrightarrow(1+\beta) \sigma\left(\xi_{v+1}, \xi_{v+2}\right) \leq(1+\beta) \sigma\left(\xi_{v}, \xi_{v+1}\right) \\
& \Longrightarrow \sigma\left(\xi_{v+1}, \xi_{v+2}\right) \leq \sigma\left(\xi_{v}, \xi_{v+1}\right)
\end{aligned}
$$

Now, we claim that

$$
\begin{gathered}
\sigma\left(\xi_{v}, \xi_{v+1}\right) \leq 2 \sigma\left(\xi_{v}, z\right) \text { or } \\
\sigma\left(\xi_{v+1}, \xi_{v+2}\right) \leq 2 \sigma\left(\xi_{v+1}, z\right) .
\end{gathered}
$$




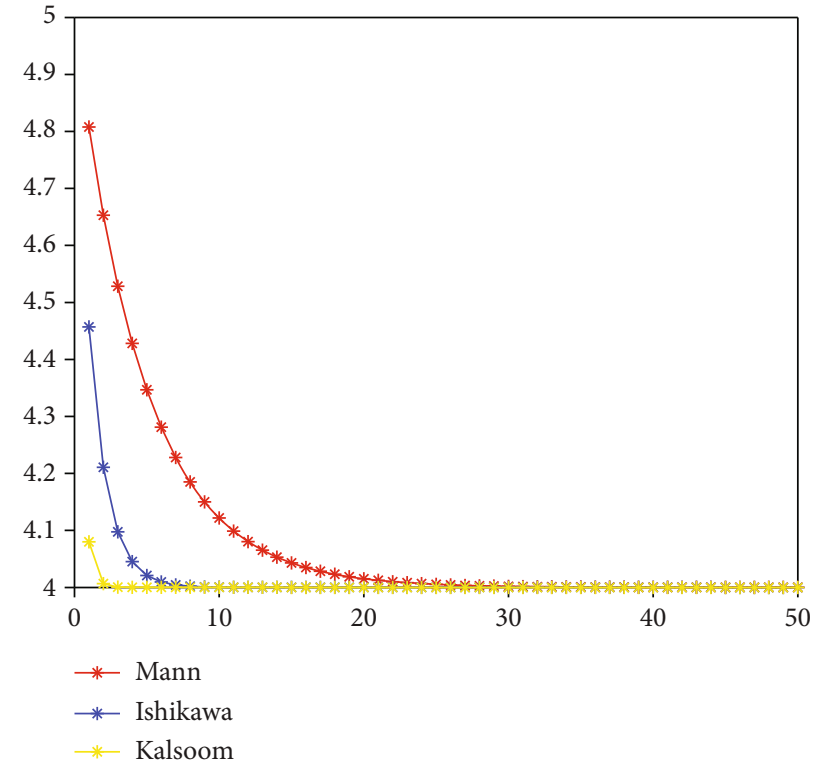

FIgURE 4: Convergence behavior of Mann, Ishikawa, and Kalsoom et al. iterations towards fixed point.

To prove this, we consider the contradiction

$$
\begin{aligned}
& 2 \sigma\left(\xi_{v}, z\right)<\sigma\left(\xi_{v}, \xi_{v+1}\right) \text { and } \\
& 2 \sigma\left(\xi_{v+1}, z\right)<\sigma\left(\xi_{v+1}, \xi_{v+2}\right) .
\end{aligned}
$$

By using triangular inequality,

$$
\begin{aligned}
\sigma\left(\xi_{v}, \xi_{v+1}\right) & \leq \sigma\left(\xi_{v}, z\right)+\sigma\left(\xi_{v+1}, z\right) \\
& <\frac{1}{2} \sigma\left(\xi_{v}, \xi_{v+1}\right)+\frac{1}{2} \sigma\left(\xi_{v+1}, \xi_{v+2}\right) \\
& <\sigma\left(\xi_{v}, \xi_{v+1}\right)
\end{aligned}
$$

which is not possible, so (102) is satisfied.

In the first case of (102),

$$
\begin{gathered}
\frac{1}{2} \sigma\left(\xi_{v}, \xi_{v+1}\right) \leq \sigma\left(\xi_{v}, z\right) \\
\frac{1}{2} \sigma\left(\xi_{v}, \mathscr{T} \xi_{v}\right) \leq \sigma\left(\xi_{v}, z\right) \\
\sigma\left(\mathscr{T} \xi_{v}, \mathscr{T} \mathrm{z}\right) \leq \sigma\left(\xi_{v}, z\right)+\beta\left\{\sigma\left(\xi_{v}, z\right)-\sigma\left(\mathscr{T} \xi_{v}, \mathscr{T} \mathrm{z}\right)\right\} \\
(1+\beta) \sigma\left(\mathscr{T} \xi_{v}, \mathscr{T} \mathrm{z}\right) \leq(1+\beta) \sigma\left(\xi_{v}, z\right) \\
\sigma\left(\mathscr{T} \xi_{v}, \mathscr{T} \mathrm{z}\right) \leq \sigma\left(\xi_{v}, z\right) .
\end{gathered}
$$

Putting lim sup on both sides,

$$
\begin{gathered}
\limsup _{v \longrightarrow \infty} \sigma\left(\mathscr{T} \xi_{v}, \mathscr{T} z\right) \leq \underset{v \longrightarrow \infty}{\lim \sup } \sigma\left(\xi_{v}, z\right) \\
\mathscr{T} z=z .
\end{gathered}
$$

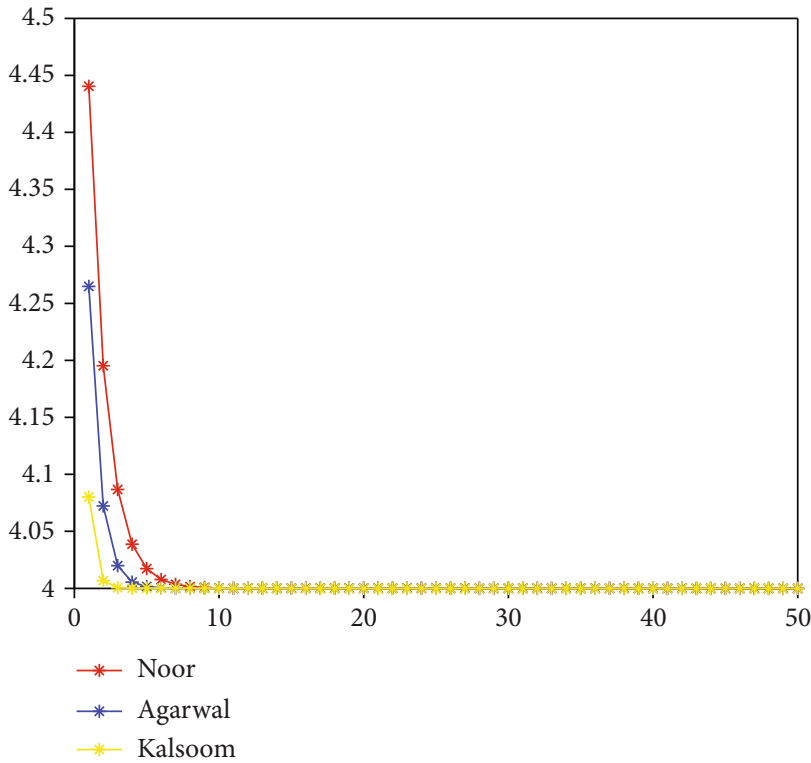

Figure 5: Convergence behavior of Noor, Agarwal, and Kalsoom et al. iterations towards fixed point.

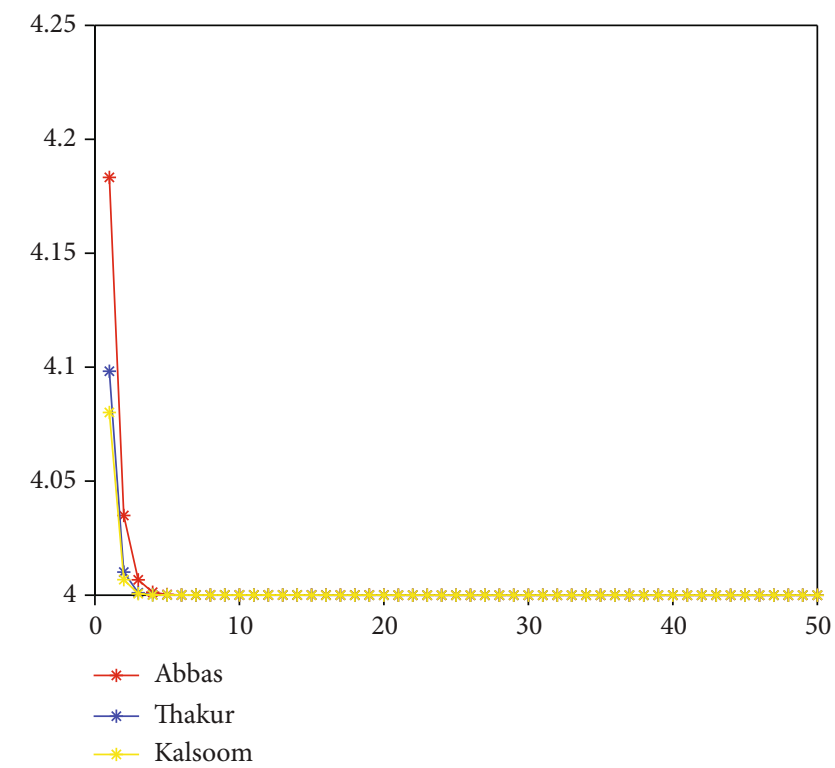

Figure 6: Convergence behavior of Abbas, Thakur, and Kalsoom et al. iterations towards fixed point.

Similarly, in the second case,

$$
\begin{gathered}
\frac{1}{2} \sigma\left(\xi_{v+1}, \xi_{v+2}\right) \leq \sigma\left(\xi_{v+1}, z\right) \\
\frac{1}{2} \sigma\left(\xi_{v+1}, \mathscr{T} \xi_{v+1}\right) \leq \sigma\left(\xi_{v+1}, z\right) \\
\sigma\left(\mathscr{T} \xi_{v+1}, \mathscr{T} \mathrm{z}\right) \leq \sigma\left(\xi_{v+1}, z\right)+\beta\left\{\sigma\left(\xi_{v+1}, z\right)-\sigma\left(\mathscr{T} \xi_{v+1}, \mathscr{T} \mathrm{z}\right)\right\} \\
(1+\beta) \sigma\left(\mathscr{T} \xi_{v+1}, \mathscr{T} \mathrm{z}\right) \leq(1+\beta) \sigma\left(\xi_{v+1}, z\right) \\
\sigma\left(\mathscr{T} \xi_{v+1}, \mathscr{T} \mathrm{z}\right) \leq \sigma\left(\xi_{v+1}, z\right) .
\end{gathered}
$$


Putting lim sup on both sides,

$$
\begin{gathered}
\limsup _{v \longrightarrow \infty} \sigma\left(\mathscr{T} \xi_{v+1}, \mathscr{T} z\right) \leq \underset{v \longrightarrow \infty}{\lim \sup } \sigma\left(\xi_{v+1}, z\right), \\
\mathscr{T} z=z .
\end{gathered}
$$

Conversely, $F(\mathscr{T}) \neq \varnothing$; then, there exists some $w \in F(\mathscr{T})$ and $\mathscr{T}^{v}(w)=w \forall v \in \mathbb{N}$; then, $\left\{T^{v}(w)\right\}$ is a constant sequence, and hence, it is bounded and this completes the proof.

Example 4. Let $\mathscr{T}: W \longrightarrow W$ where $W=[3,6]$; then, $\mathscr{T}$ is defined as

$$
\mathscr{T} \xi=\sqrt{3 \xi+4}
$$

for any $\xi \in W$; take $\alpha_{v}=0.7, \beta_{v}=0.6$, and $\gamma_{v}=0.5$. The fixed point of $\mathscr{T}$ is 4 , and take initial point as $\xi_{0}=5$. Then, $\mathscr{T}$ is monotone generalized $\beta$-nonexpansive mapping.

In Table 2, we discussed the convergence behavior of some iteration processes. It is clear that all iterations approach to 4 which is the fixed point of $\mathscr{T}$. In this case, Figures 4-6 show that Kalsoom et al. iteration process converges faster to the fixed point as compared the other iterations.

\section{Conclusion}

It concludes that we have approximated fixed point results of monotone $\alpha$ and generalized $\beta$-nonexpansive mappings in hyperbolic spaces. Moreover, we proved some numerical applications and presented the graphical representations by using different iteration processes.

\section{Data Availability}

The data used to support the findings of this study are available from the corresponding author upon request.

\section{Conflicts of Interest}

The authors declare to have no competing interests.

\section{Authors' Contributions}

All authors contributed equally and significantly in writing this article. All authors read and approved the final manuscript.

\section{Acknowledgments}

Authors are thankful to the editor and anonymous referees for their valuable comments and suggestions.

\section{References}

[1] F. E. Browder, "Nonexpansive nonlinear operators in a Banach space," Proceedings of the National Academy of Sciences of the United States of America, vol. 54, no. 4, pp. 1041-1044, 1965.
[2] D. Göhde, "Zum prinzip der Kontraktiven abbildung," Mathematische Nachrichten, vol. 30, no. 3-4, pp. 251-258, 1965.

[3] W. A. Kirk, "A fixed point theorem for mappings which do not increase distances," The American Mathematical Monthly, vol. 72, no. 9, pp. 1004-1006, 1965.

[4] A. Dehici and R. Najeh, "Some fixed point results for nonexpansive mappings in Banach spaces," Journal of Nonlinear Functional Analysis, vol. 2020, no. 1, article 36, 2020.

[5] B. Tan and S. Y. Cho, "An inertial Mann-like algorithm for fixed points of nonexpansive mappings in Hilbert spaces," Journal of Applied and Numerical Optimization, vol. 2, no. 3, pp. 335-351, 2020.

[6] A. C. Ran and M. C. B. Reurings, "A fixed point theorem in partially ordered sets and some applications to matrix equations," Proceedings of the American Mathematical Society, vol. 132, no. 5, pp. 1435-1443, 2004.

[7] J. J. Nieto and R. Rodríguez-López, "Contractive mapping theorems in partially ordered sets and applications to ordinary differential equations," Order, vol. 22, no. 3, pp. 223-239, 2005.

[8] Y. Song, K. Promluang, P. Kumam, and Y. Je Cho, "Some convergence theorems of the Mann iteration for monotone $\alpha$ -nonexpansive mappings," Applied Mathematics and Computation, vol. 287-288, pp. 74-82, 2016.

[9] B. A. Bin Dehaish and M. A. Khamsi, "Browder and Göhde fixed point theorem for monotone nonexpansive mappings," Fixed Point Theory and Applications, vol. 2016, no. 1, Article ID 20, 2016.

[10] T. Suzuki, "Fixed point theorems and convergence theorems for some generalized nonexpansive mappings," Journal of Mathematical Analysis and Applications, vol. 340, no. 2, pp. 1088-1095, 2008.

[11] K. Aoyama and F. Kohsaka, "Fixed point theorem for $\alpha$-nonexpansive mappings in Banach spaces," Nonlinear Analysis, vol. 74, no. 13, pp. 4387-4391, 2011.

[12] R. Pant and R. Shukla, "Approximating fixed points of Generalized $\alpha$-Nonexpansive mappings in Banach spaces," Numerical Functional Analysis and Optimization, vol. 38, no. 2, pp. 248-266, 2017.

[13] S. P. Pandey and D. P. Shukla, "Fixed point theorem using monotone generalised $\beta$-nonexpansive mappings in Banach space," International Journal of Innovations in Engineering and Technology (IJIET), vol. 11, no. 2, p. 2319-1058, 2018.

[14] T. C. Lim, "Remarks on some fixed point theorems," Proceedings of the American Mathematical Society, vol. 60, no. 1, pp. 179-182, 1976.

[15] B. Tan and S. Li, "Strong convergence of inertial Mann algorithms for solving hierarchical fixed point problems," Journal of Nonlinear and Variational Analysis, vol. 4, no. 3, pp. 337355, 2020.

[16] W. A. Kirk and B. Panyanak, "A concept of convergence in geodesic spaces," Nonlinear Analysis, vol. 68, no. 12, pp. 3689-3696, 2008.

[17] Y. Yao, L. Leng, M. Postolache, and X. Zheng, "Mann-type iteration method for solving the split common fixed point problem," Journal of Nonlinear and Convex Analysis, vol. 18, no. 5, pp. 875-882, 2017.

[18] Y. Yao, X. Qin, and J. C. Yao, "Projection methods for firmly type nonexpansive operators," Journal of Nonlinear and Convex Analysis, vol. 19, no. 3, pp. 407-415, 2018.

[19] F. Kohsaka and W. Takahashi, "Existence and approximation of fixed points of firmly nonexpansive-type mappings in 
Banach spaces," SIAM Journal on Optimization, vol. 19, no. 2, pp. 824-835, 2008.

[20] M. Bachar and M. A. Khamsi, "On common approximate fixed points of monotone nonexpansive semigroups in Banach spaces," Fixed Point Theory and Applications, vol. 2015, no. 1, Article ID 160, 2015.

[21] U. Kohlenbach, "Some logical metatheorems with applications in functional analysis," Transactions of the American Mathematical Society, vol. 357, no. 1, pp. 89-128, 2005.

[22] W. Takahashi, "A convexity in metric space and nonexpansive mappings. I," Kodai Mathematical Journal, vol. 22, no. 2, pp. 142-149, 1970.

[23] K. Goebel and W. A. Kirk, "Iteration processes for nonexpansive mappings," Contemporary Mathematics, vol. 21, pp. 115123, 1983.

[24] F. Itoh, "Some fixed point theorems in metric spaces," Fundamenta Mathematicae, vol. 102, no. 2, pp. 109-117, 1979.

[25] A. Kalsoom, M. Rashid, T. Sun et al., "Fixed points of monotone total asymptotically nonexpansive mapping in hyperbolic space via new algorithm," Journal of Function Spaces, vol. 2021, Article ID 8482676, p. 10, 2021.

[26] W. R. Mann, "Mean value methods in iteration," Proceedings of the American Mathematical Society, vol. 4, no. 3, pp. 506510, 1953.

[27] S. Ishikawa, "Fixed points by a new iteration method," Proceedings of the American Mathematical Society, vol. 44, no. 1, pp. 147-150, 1974.

[28] M. A. Noor, "New approximation schemes for general variational inequalities," Journal of Mathematical Analysis and Applications, vol. 251, no. 1, pp. 217-229, 2000.

[29] R. P. Agarwal, D. O'Regan, and D. R. Sahu, "Iterative construction of fixed points of nearly asymptotically nonexpansive mappings," Journal of Nonlinear and Convex Analysis, vol. 8, pp. 61-79, 2007.

[30] M. Abbas and T. Nazir, "A new faster iteration process applied to constrained minimization and feasibility problems," Matematicki Vesnik, vol. 66, pp. 223-234, 2014.

[31] B. S. Thakur, D. Thakur, and M. Postolache, "A new iterative scheme for numerical reckoning fixed points of Suzuki's generalized nonexpansive mappings," Applied Mathematics and Computation, vol. 275, pp. 147-155, 2016.

[32] B. E. Rhoades, "Some fixed point iteration procedures," International Journal of Mathematics and Mathematical Sciences, vol. 14, no. 1, 16 pages, 1991. 